\title{
PRUEBA DE UNA DIETA CONCENTRADA Y SUPLEMENTADA CON ELEMENTOS NATURALES PARA PRIMATES NEOTROPICALES CAUTIVOS
}

\section{TEST A CONCENTRATED AND SUPPLEMENTED DIET WITH NATURAL ELEMENTS FOR NEOTROPICALES PRIMATES IN CAPTIVITY}

\author{
FAJARDO-PATIÑO, ALIRIO ${ }^{1^{*}}$ MSc., DE LA OSSA, V. JAIME ${ }^{2^{* *}}$ Dr. \\ ${ }^{1^{\star}}$ Fundación George Dahl, Colombia. ${ }^{2^{\star \star}}$ Universidad de Sucre, Facultad de \\ Ciencias Agropecuarias Grupo Biodiversidad Tropical, Colombia. \\ Correspondencia: *afajardop@gmail.com ${ }^{\star *}$ jaimedelaossa@yahoo.com
}

\section{Resumen}

Recibido: 10-01-2014; Aceptado: 26-06-2014.

En el presente estudio se ensayó una dieta balanceada complementada con frutas y presas vivas para el mantenimiento en condiciones de semicautividad y cautividad de una colonia de primates que contó con individuos de las siguientes especies: Aotus spp., Saguinus oedipus y Cebuella pygmaea. Se elaboró una galleta previo análisis de los requerimientos nutricionales de primates no humanos neotropicales conducidos en otros centros de manejo e investigación pertinentes. Se evidencia el consumo de preferencial de insectos y lagartos como proteína de origen animal, la dieta ofrecida y con un porcentaje de proteína ubicada en el nivel mínimo sugerido para colonias cautivas de primates tuvo aceptación y permitió mantener en buen estado corporal a los animales sujetos a experimentación. Los resultados muestran que es factible ajustar una dieta con elementos disponibles en la región y fácilmente accesibles, lo cual es de gran valor para el manejo de estas especies de primates neotropicales en condiciones controladas de semicautiverio y cautiverio.

Palabras clave: primates neotropicales, dieta balanceada, cautiverio, Colosó, Sucre, Colombia.

\begin{abstract}
Presently study was tested a balanced diet supplemented with fruits and alive preys for the maintenance of a primates captivity colony hat had individuals of the following species: Aotus spp., Saguinus oedipus and Cebuella pygmaea. A cookie was elaborated previous analysis of the nutritional requirements for Neotropical non human primates driven in other research centers and pertinent investigations. The preferential consumption were insects and lizards like protein of animal origin, the offered diet with a protein percentage located in the minimum level suggested for captive colonies of primates it had acceptance and it allowed to maintain in good corporal state to the animals subject to experimentation. The results show that it is feasible to adjust a diet with
\end{abstract}


available elements in the region and easily accessible, that which is of great value for the handling of these species of Neotropical primates under controlled semi captivity and captivity conditions.

Key words: Neotropical Primates, balanced diet, captivity, Colosó, Sucre, Colombia.

\section{Introducción}

En su medio natural la mayoría de la especies de primates consumen una larga variedad de presas y plantas (RICHARDS, 1985); al hacer referencia al consumo de plantas y sus partes, se demuestra en diversos estudios que su consumo no es realizado al azar y que existe una marcada preferencia (CHIVERS, 1998), que se relaciona con factores nutricionales y con contenido tóxico de ciertas especies vegetales en particular (BARTON y WHITEN, 1994), depende también de la disponibilidad temporal y espacial, así mismo influyen en la selección de la dieta factores como talla corporal, morfología digestiva y adaptaciones evolutivas (LASKA et al., 2000).

De forma particular y con relación a medio natural la selección de alimento en Callitrichidae muestra preferencia por frutas, insectos y en algunas especies exudados vegetales, siendo el consumo de insectos un punto de gran importancia (GARBER, 1992); por su parte en Cebidae las variaciones son mayores entre especies, pueden ser literalmente folívoros como Alouatta seniculus o generalistas oportunistas como Cebus capucinus, en todo caso incluyen vegetales y animales en su dieta (HERNÁNDEZ-CAMACHO y COOPER, 1976).

Como norma para el manejo de primates en cautividad se establece que la dieta a ofrecer debe ser palatable, nutricionalmente adecuada y libre de contaminación y estar de acuerdo con los requerimientos de la especie; igualmente se plantea que el agua a proveer debe ser limpia, constante y no debe producir encharcamientos dentro del encierro para evitar la diseminación de enfermedades (IPS, 2006). La formulación de una dieta que supla los requerimientos energéticos y proteínicos mínimos es indispensable para la realización de adecuadas funciones metabólicas, es a su vez uno de los mayores inconvenientes para el establecimiento de colonias de primates en cautiverio (BUCHANAN et al., 2002).

Ensayos de alimentación conducidos en cautiverio con primates del nuevo mundo han sido desarrollados por diversos autores (NRC, 1978; AUSMAN et al., 1979; AUSMAN et al., 1985; CLAPP y TARDIF, 1985; RICHARD, 1985; POOLE, 1990; CHARNOCK et al., 1992; HANNAFORD, 1996; LASKA et al., 
1996; POWER y OFTEDAL, 1996; ELY et al., 1998; LASKA et al., 1998; LASKA et al., 1999; MILTON, 1998; TARDIF et al., 1998; CORREA et al., 2000; LASKA et al., 2000; GORE et al., 2001), que han demostrado los beneficios que dietas a base de elementos naturales y fácilmente disponibles tienen para su uso en animales cautivos.

En el presente trabajo se experimentó con tres colonias de primates neotropicales cautivos y semicautivos: Aotus spp., Saguinus oedipus y Cebuella pygmaea, se ofreció regularmente una dieta balanceada cuya presentación era en forma de galleta horneada y posteriormente hidratada con agua azucarada y acompañada de porciones de frutas frescas, se suplementó semanalmente con una solución multivitamínica y mineral, y de dos a tres veces por semana, a voluntad, se suministraron insectos y lagartos vivos.

\section{Materiales y métodos}

Ubicación del área: La Estación Primates del INDERENA (entidad liquidada, cuyas funciones fueron asumidas regionalmente por la Corporación Autónoma Regional de Sucre-CARSUCRE-), ubicada a los $9^{\circ} 31^{\prime} 48.77^{\prime \prime} \mathrm{N} ; 7^{\circ} 21^{\prime} 5.57^{\prime \prime} \mathrm{W}$, distante a $5 \mathrm{~km}$ del perímetro urbano del casco municipal de Colosó, en inmediaciones de la Reserva Forestal Protectora Serranía de Coraza Montes de María, departamento de Sucre, costa Caribe, Colombia (Figs. 1 y 2).

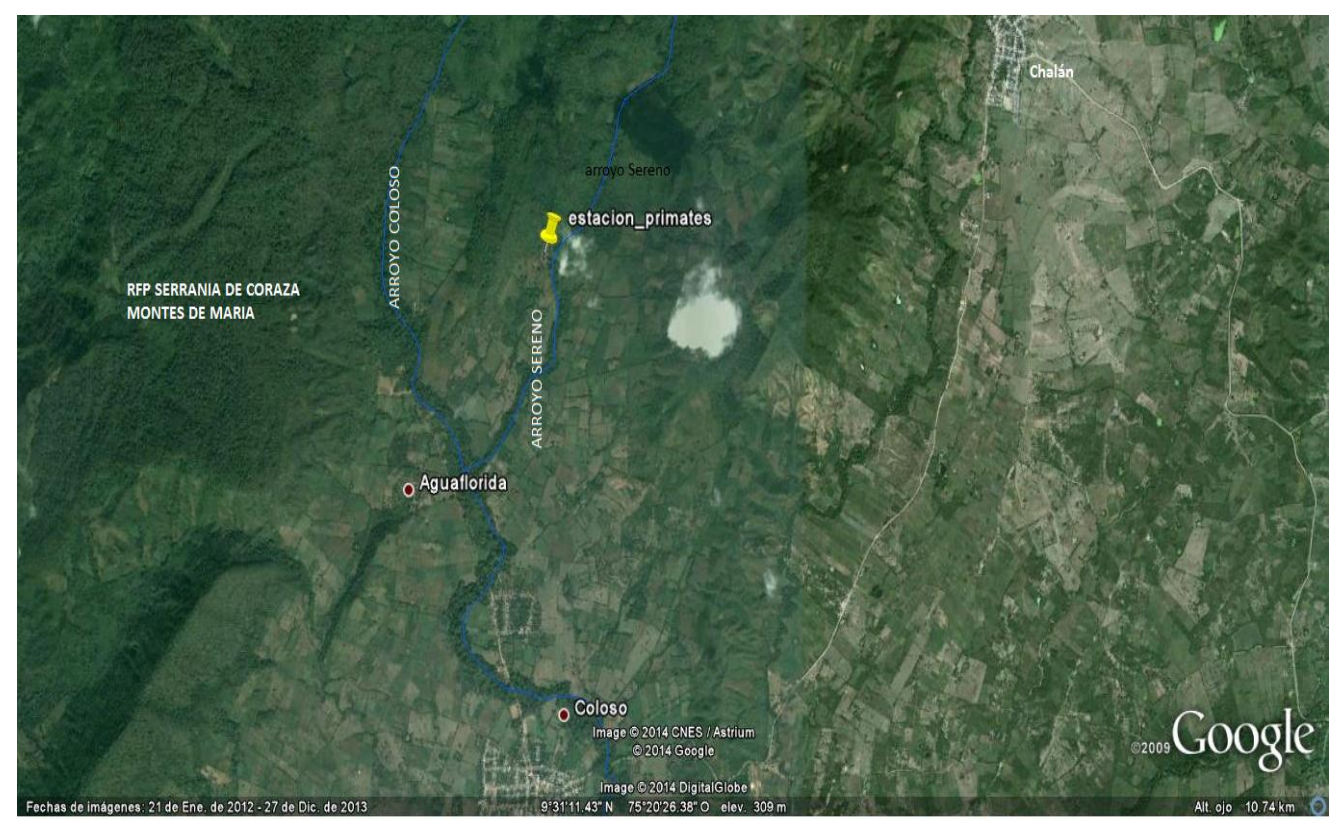

Figura 1. Ubicación de la Estación Primates, Colosó, Sucre (Google Earth, Free, versión 5.0) 


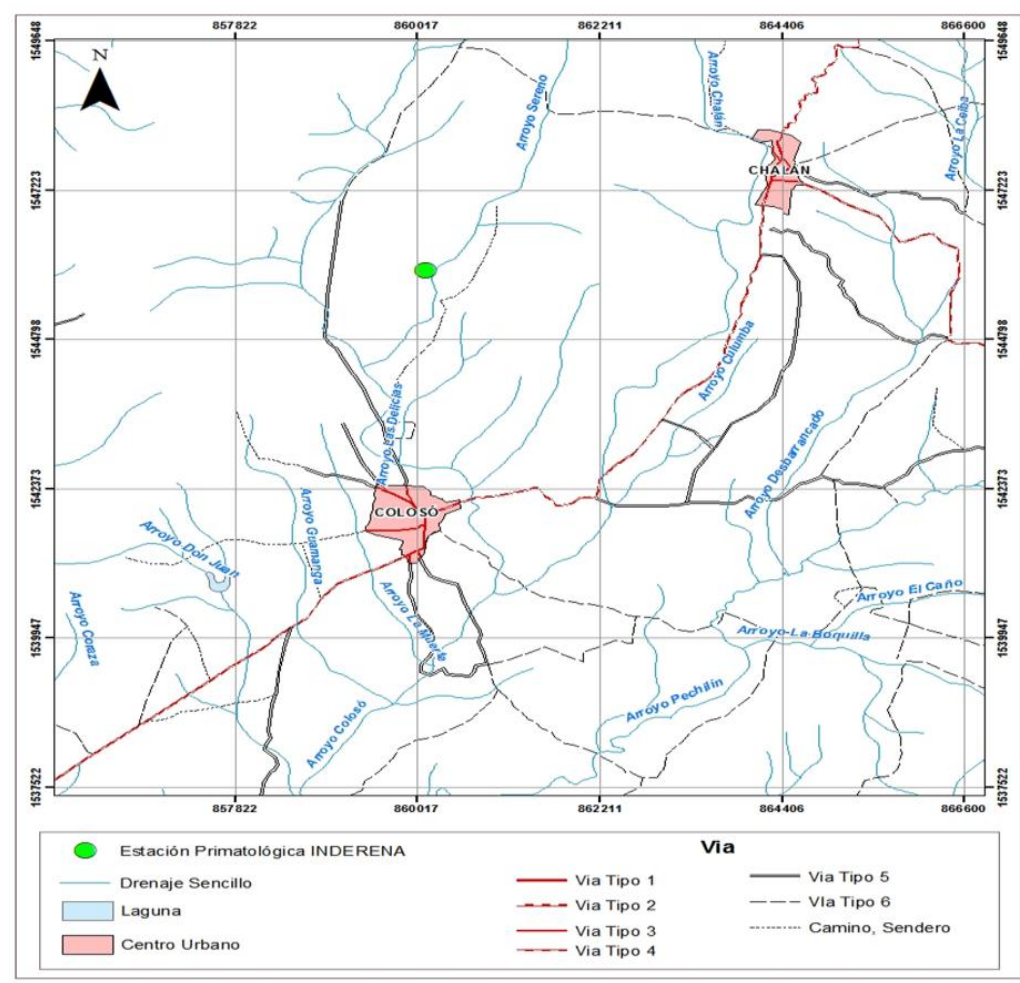

Figura 2. Localización Estación Primatológica INDERENA

Infraestructura: Los ensayos fueron conducidos en cuatro galpones para el mantenimiento de primates cautivos, discriminados, así: 2 unidades de cautividad, con área individual de $6 \times 16 \mathrm{~m}$, altura central de 3,7 m y 2,4 m de altura en los flancos, construidos con estructuras modulares prefabricadas, provistos de mallas de angeo mosquitero en sus paredes exteriores, interiormente cada galpón posee 24 jaulas de $0,7 \times 0,7 \times 2 \mathrm{~m}$, con una caja refugios en la parte externa superior frontal (Fig. 3).

Dos unidades de semicautividad, con las mismas características estructurales descritas, sin jaulas interiores y perímetro total y parte media del techado forrado en malla eslabonada de alambre calibre 14 y $1^{\prime \prime}$ de ojo, con la otra mitad del techo cubierto por láminas de asbesto cemento (Fig. 4).

Adicionalmente cada unidad contó con los comederos, bebederos, perchas y abrigos necesarios y pertinentes a las necesidades de cada una de las especies manejadas. 


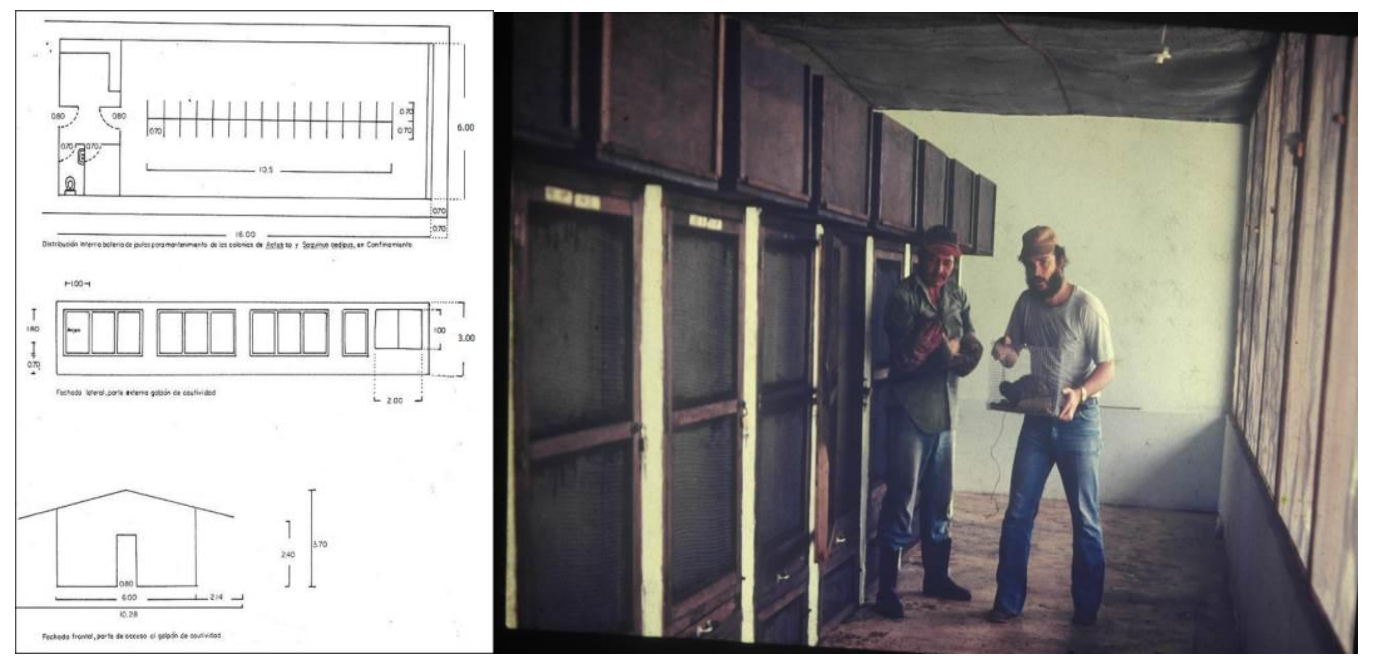

Figura 3. Plano y aspecto de la estructura interior de galpón de cautiverio para Aotus spp., Saguinus oedipus y Cebuella pygmaea

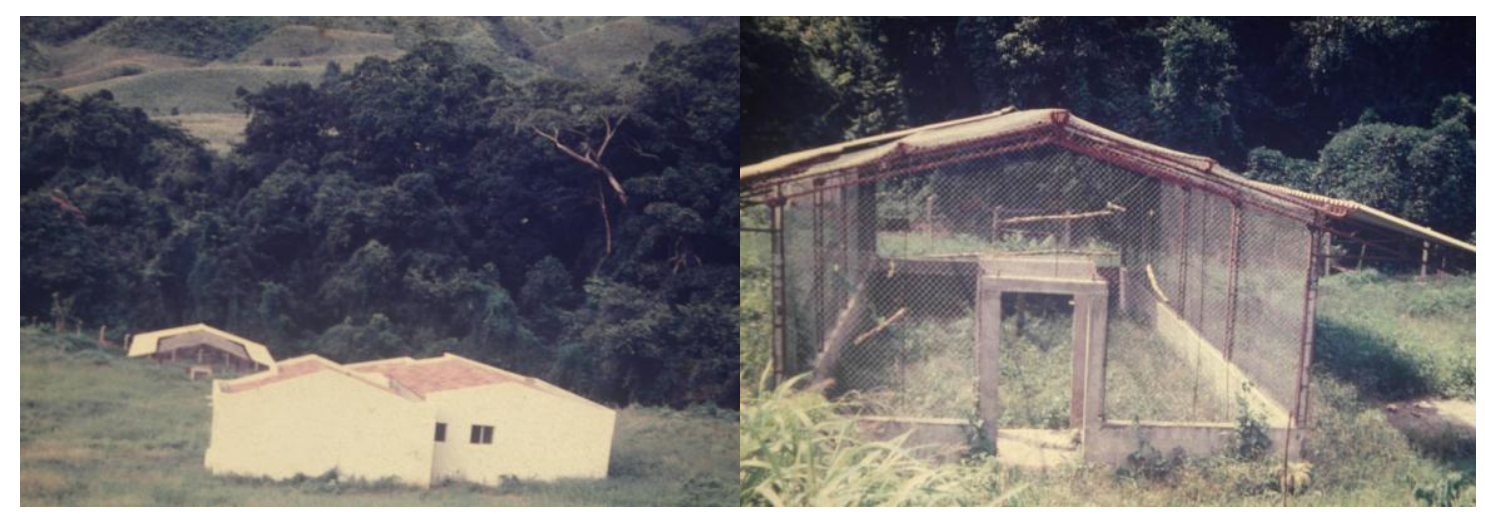

Figura 4. Vista de galpón de semicautiverio para mantenimiento de Saguinus oedipus, a la izquierda al fondo galpón cerca del bosque, derecha primer plano del mismo galpón

Colonia experimental: Estuvo constituida por 8 ejemplares de Saguinus oedipus ( 2 machos y 6 hembras), 5 de Cebuella pygmaea ( 2 machos y 3 hembras) (Fig. 5), todos los especímenes eran provenientes de decomisos realizados por el Instituto Nacional de los Recursos Naturales Renovables y del Medio Ambiente (INDERENA), y 27 Aotus spp, correspondientes a: $A$. nancymai (5 machos y 6 hembras), $A$. nigriceps (3 machos y 5 hembras), $A$. vociferans (4 machos y 4 hembras) procedentes de la colonia experimental que sirvió de base para la investigación en malaria del Dr. Manuel Elkin Patarroyo en Leticia, Amazonas, Colombia (1984-1985).

Los 27 Aotus spp. fueron recibidos en mal estado de salud, externamente se evidenciaban lesiones abdominales sobreelevadas, con solución de continuidad por pérdida de sustancia, con coloración violácea; bajo peso y diversas laceraciones cutáneas, las que permitían inferir un deficiente manejo 
de estos sujetos experimentales y la ausencia de adecuadas medidas de bienestar animal (Fig. 6).

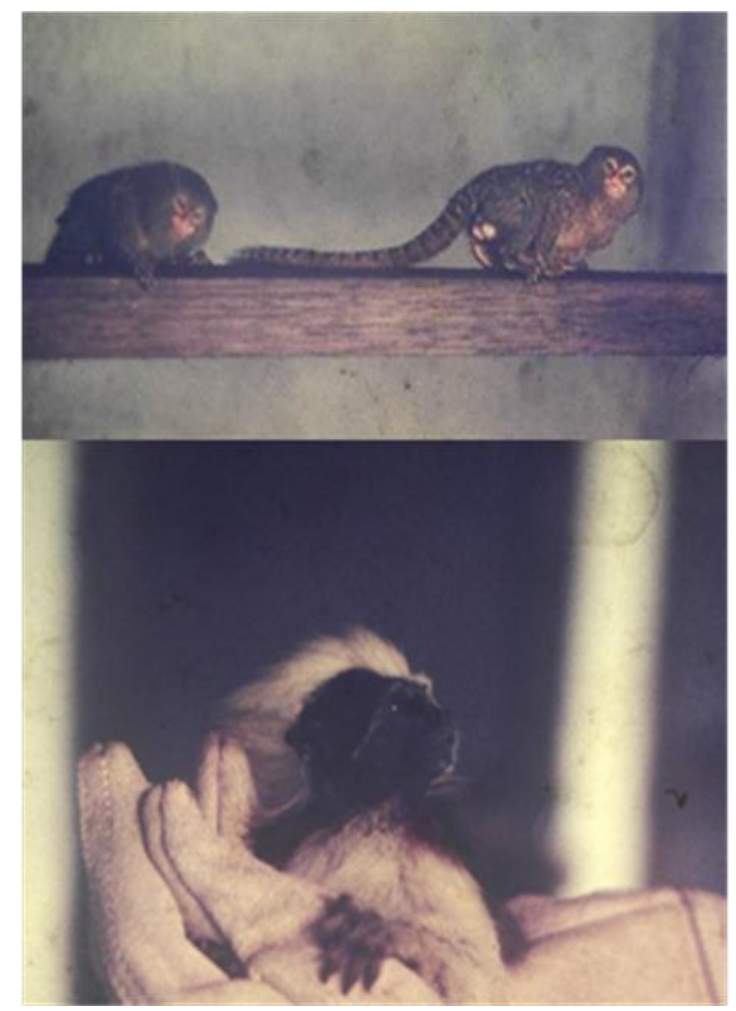

Figura 5. Ejemplares manejados en la Estación Primates. Cebuella pygmaea (superior), Saguinus oedipus (inferior)

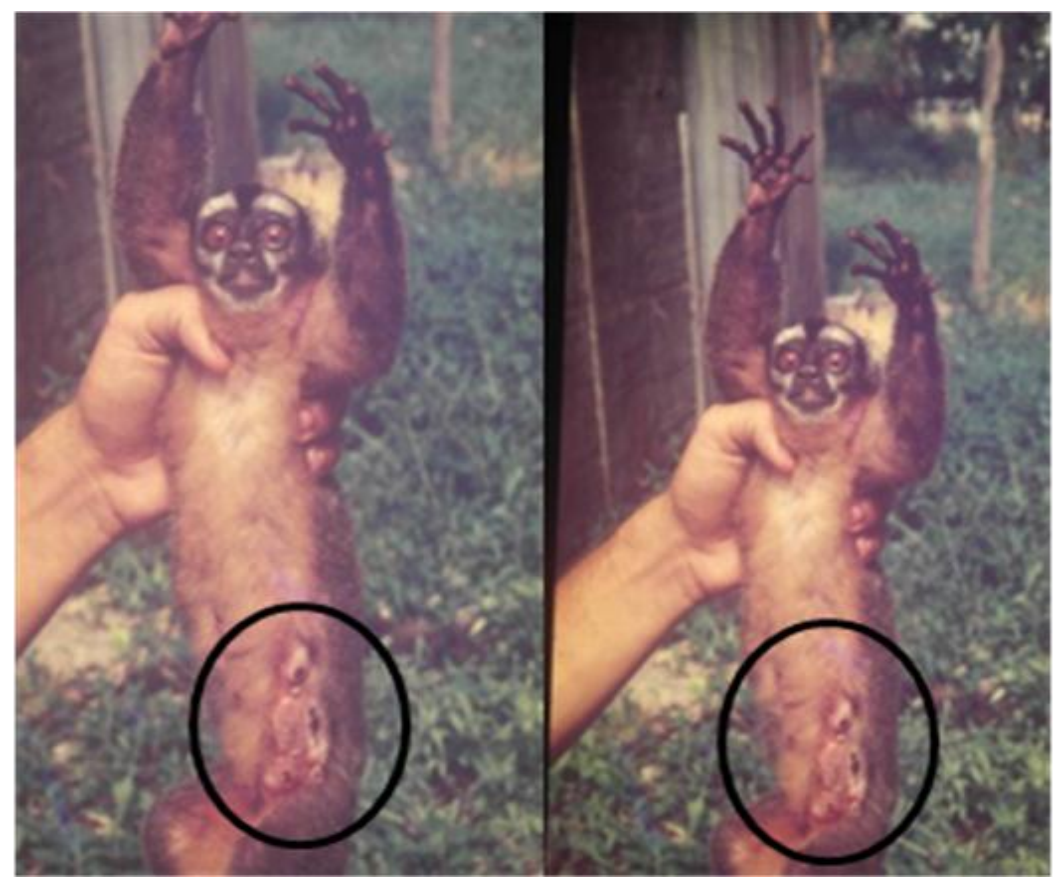

Figura 6. Aotus spp. Con evidencia de lesiones abdominales 
Dieta experimental: Se utilizó una ración balanceada, siguiendo las recomendaciones propuestas por NAPKA y MORIN (1979) para ese tipo de animales, cuya presentación era en forma de galleta horneada, se usaron como experiencia base los resultados observados en el manejo alimenticio de las colonias de primates cautivos del Centro de Primatología de lquitos, Perú, y del Laboratorio de Campo Ernesto Samper Sordo del Instituto Nacional de Salud, Colombia, en donde se desarrollaban trabajos similares. Las galletas horneadas poseían una consistencia semidura y su presentación en forma de porciones de $2 \times 2 \mathrm{~cm}$, con peso medio de 11,62 g. La dieta estuvo constituida por una porción de la mencionada galleta, porción de frutas de cosecha y agua azucarada. Se suplementó una vez por semana con una solución multivitamínica y mineral, y de dos a tres veces por semana con una ración de proteína animal. La composición de la galleta se presenta en la Tabla 1.

Se llevó a cabo un registro diario del volumen consumido, porcentaje de aceptación de cada porción del alimento ofrecido y estado de salud aparente de los animales. La determinación del porcentaje consumido se calculo teniendo en cuenta el peso del alimento ofrecido y el peso del alimento sobrante. Se dieron dos raciones diarias a las $06: 00$ y las 16:00 horas. Los pesajes de los animales se hicieron semanalmente.

Tabla 1. Ingredientes de la galleta suministrada $(\mathrm{PC}=$ proteína cruda)

\begin{tabular}{llc}
\hline \multicolumn{1}{c}{ Ingredientes } & Porcentaje (\%) \\
\cline { 2 - 3 } & Leche en polvo entera & 20 \\
Harina de trigo & 20 \\
Harina de avena & 10 \\
Harina de frijol rojo & 15 \\
Harina de maíz amarillo & 9 \\
Harina de cebada & 3 \\
Huevos & 13 \\
Levadura & 1,5 \\
Azúcar & 5 \\
Aceite vegetal & 2,5 \\
Sal yodada & 0,75 \\
Aditivos * & 0,25 \\
\cline { 2 - 3 } Total & $\mathbf{1 0 0}$ \\
\cline { 2 - 3 } * Gluconato de & Calcio 2 g, esencia de frutas $32 \mathrm{ml}$, esencia de vainilla $16 \mathrm{ml}$.
\end{tabular}

La galleta presentó aspecto solido, color amarillo, olor característico y agradable, macroscópicamente sin partículas extrañas. El análisis fisicoquímico se presenta en la Tabla 2.

La composición porcentual del suplemento multivitamínico y mineral se muestra en la Tabla 3, en la Tabla 4 se indica la composición detallada del aporte multivitamínico y mineral. 
Tabla 2. Composición química de la galleta

\begin{tabular}{lc}
\hline \multicolumn{1}{c}{ Contenido } & Porcentaje (\%) \\
\hline Sólidos totales & 92,26 \\
Humedad & 7,74 \\
Cenizas & 2,66 \\
Cloruros $(\mathrm{NaCl})$ & 1,19 \\
Proteína & 14,42 \\
Carbohidratos & 66,57 \\
Grasa & 7,34 \\
Fibra & 1,27 \\
$\mathrm{NDT}^{*}$ & 85 \\
pH $\left(\right.$ Sol. $10 \%$ a $\left.20{ }^{\circ} \mathrm{C}\right)$ & 5,82 \\
Peso total de la muestra $(\mathrm{g})$ & 92,40 \\
Peso medio galleta $(\mathrm{g})$ & 11,62 \\
\hline *Valor calculado (nutrientes digestibles totales)
\end{tabular}

Tabla 3. Composición del suplemento multivitamínico y mineral

\begin{tabular}{lc}
\hline Compuestos & Porcentaje \\
\hline Nestum $\AA$ & 4,77 \\
Procavit-B® & 4,77 \\
Azúcar & 5,61 \\
Pediavit $\AA$ & 0,56 \\
Redoxon $\AA$ & 0,11 \\
Agua & 84,18 \\
\hline
\end{tabular}

Tabla 4. Discriminación proximal de los componentes aportados por el suplemento multivitamínico y mineral

\begin{tabular}{lcc}
\hline \multicolumn{1}{c}{ Componentes } & Cantidad total & $\begin{array}{c}\text { Cantidad } \\
\text { /ind/semana }\end{array}$ \\
\hline Proteínas & $29,4 \mathrm{~g}$ & $882 \mathrm{mg}$ \\
Carbohidratos & $17 \mathrm{~g}$ & $510 \mathrm{mg}$ \\
Vit A & $14,875 \mathrm{UI}$ & $446,25 \mathrm{Ul}$ \\
Vit. D & $2.720 \mathrm{Ul}$ & $81,6 \mathrm{UI}$ \\
Vit. D3 & $595 \mathrm{Ul}$ & $17,85 \mathrm{Ul}$ \\
Vit. B1 & $88,51 \mathrm{mg}$ & $2,65 \mathrm{mg}$ \\
Vit. B2 & $9,94 \mathrm{mg}$ & $0,3 \mathrm{mg}$ \\
Vit. B6 & $8,3 \mathrm{mg}$ & $0,25 \mathrm{mg}$ \\
Vit. B12 & $1,66 \mathrm{mcg}$ & $0,05 \mathrm{mcg}$ \\
Vit. C & $2 \mathrm{~g}$ & $60 \mathrm{mg}$ \\
Vit. E & $7,31 \mathrm{mg}$ & $0,22 \mathrm{mg}$ \\
Vit. PP & $85 \mathrm{mg}$ & $2,55 \mathrm{mg}$ \\
Vit B3, Nicotinamida & $71 \mathrm{mg}$ & $2,13 \mathrm{mg}$ \\
Pantotenato de Calcio & $35,77 \mathrm{mg}$ & $1,07 \mathrm{mg}$ \\
Fosfato de calcio dibásico & $5,1 \mathrm{~g}$ & $152 \mathrm{mg}$ \\
Sulfato ferroso & $1,7 \mathrm{~g}$ & $51 \mathrm{mg}$ \\
Gluconato de calcio & $1 \mathrm{~g}$ & $30 \mathrm{mg}$ \\
Hierro elemental & $5 \mathrm{mg}$ & $0,15 \mathrm{mg}$ \\
Sales minerales & $2,975 \mathrm{~g}$ & $89 \mathrm{mg}$ \\
Grasa & $1,275 \mathrm{~g}$ & $38 \mathrm{mg}$ \\
\hline
\end{tabular}

La fruta ofrecida a toda la colonia experimental de primates estuvo de acuerdo con la disponibilidad de la cosecha, las más usadas, dada su fácil consecución, fueron: Carica papaya (papaya), Musa paradisiaca (banano), Mangifera indica 
(mango), Psidium guajava (guayaba), así mismo en el agua de bebida se le adicionaban gotas de cítricos: Citrus limon (limón) y Citrus sinensis (naranja).

Los insectos ofrecidos fueron: Lepidoptera (mariposas y polillas), Odonata (libélulas) y Orthoptera (Grillos), en cuanto a los lagartos, se suministraron especímenes vivos de: Anolis auratus (camaleón), Cnemidophorus lemniscatus (lobito), Ameiva ameiva (lobo) y Gonatodes albogularis (machorrito).

\section{Resultados y discusión}

En este estudio se buscó configurar una dieta general que sirviera para el mantenimiento en cautiverio de varias especies de primates neotropicales que supliera los requerimientos nutricionales básicos y que a la vez fuera de bajo costo económico, de fácil preparación y que siempre contara con una alta y permanente disponibilidad de materias primas.

En las Tablas 5, 6 y 7, se presentan los resultados de la dieta ofrecida y volumen consumido por especie.

Tabla 5. Dieta y volumen consumido por Aotus spp. (A. nancymai, A. nigriceps, A. vociferans)

\begin{tabular}{llc}
\hline \multicolumn{1}{c}{ Ración } & \multicolumn{1}{c}{ Composición } & Peso $\mathbf{( g )}$ \\
\hline Matutina & Porción de fruta fresca madura & 60 \\
& Agua fresca & Ad libitum \\
& & \\
\hline Vespertina & Porción de fruta fresca madura & 40 \\
& Galleta & 12,7 \\
& Agua azucarada $(0,1 \mathrm{~g} / \mathrm{ml})$ & 10 \\
\hline Suplemento multivitamínico y mineral & 1 vez/semana & 60 \\
Proteína de origen animal & $2-3$ veces/semana & 40 \\
\hline Consumo diario/ind. & & 122,7 \\
\hline
\end{tabular}

Tabla 6. Dieta y volumen consumido por Saguinus oedipus

\begin{tabular}{llc}
\hline \multicolumn{1}{c}{ Ración } & \multicolumn{1}{c}{ Composición } & Peso $(\mathbf{g})$ \\
\hline Matutina & Porción de fruta fresca madura & 50 \\
& Agua azucarada $(0,1 \mathrm{~g} / \mathrm{ml})$ & 10 \\
& Galleta & 6,4 \\
& Agua fresca & Ad libitum \\
\hline Vespertina & Porción de fruta fresca madura & 50 \\
\hline Suplemento multivitamínico y mineral & 1 vez/semana & 60 \\
Proteína de origen animal & $2-3$ veces/semana & 40 \\
\hline Consumo diario/indo. & & 116,4 \\
\hline
\end{tabular}


Tabla 7. Dieta y volumen consumido por Cebuella pygmaea

\begin{tabular}{llc}
\hline \multicolumn{1}{c}{ Ración } & \multicolumn{1}{c}{ Composición } & Peso $(\mathbf{g})$ \\
\hline Matutina & Porción de fruta fresca madura & 15 \\
& Agua azucarada $(0,1 \mathrm{~g} / \mathrm{ml})$ & 5 \\
& Galleta & 3,8 \\
& Agua fresca & Ad libitum \\
\hline Vespertina & Porción de fruta fresca madura & 10 \\
\hline Suplemento multivitamínico y mineral & 1 vez/semana & 25 \\
Proteína de origen animal & $2-3$ veces/semana & 30 \\
\hline Consumo diario/indo. & & 33,8 \\
\hline
\end{tabular}

Durante el primer periodo el alimento suministrado no tuvo mayor aceptación, especialmente la galleta, concentrándose la ingestión básicamente en la fruta y la proteína animal. En la Tabla 8 se presentan los registros finales de aceptación de la dieta ofrecida.

Tabla 8. Porcentaje de aceptación de la dieta

\begin{tabular}{lcccc}
\hline \multirow{2}{*}{ Especie } & \multicolumn{4}{c}{ \% de aceptación } \\
\cline { 2 - 5 } & Galleta & Fruta & Proteína animal & Suplemento \\
\hline Aotus spp. & 92,5 & 95 & 100 & 95 \\
Saguinus oedipus & 55 & 98 & 100 & 100 \\
Cebuella pygmaea & 43 & 70 & 100 & 100 \\
\hline
\end{tabular}

Existe relación significativa en cuanto al peso promedio de los individuos y el consumo diario de alimento ofrecido, siendo similar entre Saguinus oedipus y Cebuella pygmaea, pero diferenciándose notablemente de la relación existente para Aotus spp. $(p<0,05)$ (Tabla 9). La relación hallada para Aotus spp. En este estudio es baja en comparación con los registros de MERRIT (1976).

Tabla 9. Relación por especie entre peso medio y consumo diario de alimento

\begin{tabular}{lccc}
\hline Especie & $\begin{array}{c}\text { Peso medio } \\
\text { individuo } \mathbf{( g )}\end{array}$ & $\begin{array}{c}\text { Ración diaria } \\
\text { consumida }(\mathbf{g})\end{array}$ & $\begin{array}{c}\text { Relación consumo diario: } \\
\text { peso individuo }\end{array}$ \\
\hline Aotus spp. & 700 & 137 & $1: 5$ \\
Saguinus oedipus & 332 & 130,64 & $1: 2,8$ \\
Cebuella pygmaea & 85 & 41,65 & $1: 2$ \\
\hline
\end{tabular}

Aotus spp. en su medio natural consume frutos (HERNÁNDEZ y COOPER, 1976). Además de frugívoros se les describe como consumidores de follaje suplementado con huevos de aves, gusanos e insectos (HLADIK et al., 1971; RATHBUN y GACHE, 1977; WRIGTH, 1978). En cautividad aceptan bien frutos, peletizados, cereales, semillas, verduras y huevos cocidos (MERRIT, 1976; CASTRO y MONTOYA, 1980; DIXON, 1982; UMAÑA et al., 1984). Aotus es el taxón más adaptado que se tiene dentro de esta colonia de primates manejados en captividad, con un marcado consumo de todos los alimentos ofrecidos. 
Debido a observaciones previas de consumo incidental de lagartos e insectos que ingresaban libremente a los galpones de semicautiverio y a registros pertinentes sobre alimentación en medio natural (HERNÁNDEZ y COOPER, 1976; NEYMAN, 1976; SOINI, 1982), se tomó la decisión de ofrecer en la dieta lagartos e insectos, que son ampliamente apetecidos por estos primates. Cuando están en parejas el suministro de los insectos provoca fuertes interacciones, lo que hace que sea necesario alimentar al mismo tiempo de forma rápida, este comportamiento de competencia por alimento no fue observado ni con la galleta ni con la fruta. El consumo de suplemento líquido es escaso, concordando con lo descrito por BATES (1863) y SANDERSON (1957), quienes señalan que el consumo de agua en cautividad es raro.

Saguinus oedipus consume básicamente frutos e insectos (HERNÁNDEZ y COOPER, 1976). NEYMAN (1976) registra en su alimentación: frutos de árboles, enredaderas y epifitas, rebrotes, hojas, insectos y ocasionalmente anuros. Observaciones de esta investigación en medio natural indican que la depredación de lagartos es alta, de igual manera se registró el consumo de exudados de varias especies arbóreas de la zona, como Manilkara sp. En cautiverio muestran gran aceptación de alimentos peletizados, frutos, verduras (BRAND, 1971; KIRKWOOD, 1983; KIRKWOOD et al., 1983). En el presente estudio mostraron aceptación de insectos, lagartos, frutas y suplemento, la galleta fue moderadamente ingerida. El consumo de insectos se referencia como una adaptación alimenticia primaria para los Callitrichidae (GARBER, 1992) también se registra la ingestión de exudados (TERBORGH, 1983; ROSENBERGER, 1992).

Cebuella pygmaea, se mostró inicialmente reacio a consumir la fruta y la galleta, después de no suministrárseles ningún tipo de alimento acceden a ingerirlos, aún así no son ítem alimentares de su apetencia total, prefieren la proteína animal y el suplemento líquido. HERNÁNDEZ y COOPER (1976) señalan que esta especie posee una de las dietas más especializadas entre los primates neotropicales, durante una revisión de contenidos estomacales hallaron savia gelatinosa, insectos finamente macerados y evidencias de pulpa de frutas. SOINI (1977) describe a que C. pygmaea como consumidor de exudados vegetales, invertebrados y ocasionalmente recurre al consumo de pequeñas cantidades de frutas. En el presente trabajo se muestra una marcada preferencia por el suplemento líquido azucarado así como por la proteína de origen animal representada por insectos y lagartos. El consumo de saurios en su medio natural se presenta, siendo sus potenciales presas algunos lagartos de hábitos arborícolas, esto parece confirmarse por la avidez con que los consumen (FAJARDO, 1988). Mostró poca apetencia por la galleta y en algunos casos por la fruta. Igualmente como Callitrichidae DIGBY (1998) y 
TOWNSEND, (1999) registran consumo de ranas, lagartos, polluelos y huevos, lo cual coincide con los hallados en este estudio.

En cuanto al porcentaje de proteína se calcula entre 12 al 22\% (NICKEL y HEYMANN, 1996), se ha hallado que cuando los niveles de proteína en la dieta son menores al 6\% algunos Callitrichidae practican escatofagia (FLURER y ZUCKER, 1989). En este estudio se trabajo con un porcentaje de proteína cercano al valor mínimo pero superior a 7,3\% recomendado como dieta de mantenimiento en cautiverio (FLURER y ZUCKER, 1989) y los resultados en cuanto a incremento de biomasa y suceso reproductivos fueron satisfactorios (Tabla 10).

Tabla 10. Porcentaje de variación de peso corporal por especie

\begin{tabular}{lcccc}
\hline Especie & $\begin{array}{c}\text { Peso medio } \\
\text { inicial (g) }\end{array}$ & $\begin{array}{c}\text { Peso medio } \\
\text { final (g) }\end{array}$ & $\begin{array}{c}\text { \% ganancia } \\
\text { de peso }\end{array}$ & $\begin{array}{c}\text { Eventos } \\
\text { reproductivos }\end{array}$ \\
\hline Aotus spp. & 640 & 700 & 9,37 & 7 \\
Saguinus oedipus & 332,5 & 332,8 & 0 & 1 \\
Cebuella pygmaea & 73 & 85 & 16,44 & 1 \\
\hline
\end{tabular}

Los resultados muestran que es factible ajustar una dieta con elementos disponibles en la región y fácilmente accesibles, lo cual es de gran valor para el manejo de estas especies de primates neotropicales en cautiverio. Esta dieta además de poseer una buena calidad biológica permite mantener un óptimo nivel de seguridad alimentaria para los animales cautivos, como se demuestra en el presente trabajo.

\section{Referencias}

AUSMAN, L.M.; GALLINA, D.L.; R.J. NICOLOSI. 1985. Nutrition and metabolism of the squirrel monkey. pp. 349-378. En: Rosenblum, A.L.; Coe, C.L. (eds.). Handbook of Squirrel Monkey Research. Plenum Press, New York, NY.

AUSMAN, L.M.; GALLINA, D.L.; SAMONDS, K.W.; HEGSTED, D.M. 1979. Assessment of the efficiency of protein utilization in young squirrel and macaque monkeys. Am. J. Clin. Nutr. 32:1813-1823.

BARTON, R.A.; WHITEN, A. 1994. Reducing complex diets to simple rules: Food selection by olive baboons. Behav. Ecol. Sociobiol. 35: 283-293.

BATES, H.W. 1963, The naturalist on the River Amazons. John Murray, London.

BRAND, H.M. 1971. Husbandry and breeding of newly - stablesh colony of cotton-top tamarins (Saguinus oedipus). Lab. Sci. Sci. 15:7-11. 
BUCHANAN-SMITH, HM.; SHAND, C.; KEITH MORRIS, K. 2002. Cage Use and Feeding Height Preferences of Captive Common Marmosets (Callithrix $j$. jacchus) in Two-Tier Cages. Journal of Applied Animal Welfare Science 5(2), 139-149.

CASTRO, R., MONTOYA, C. 1980. Investigaciones en cautiverio de Aotus trivirgatus trivirgatus. IVITA. Iquitos, Perú.

CHARNOCK, J.S.; ABEYWARDENA, M.Y.; POLETTI, V.M.; MCLENNAN, P.L. 1992. Difference in fatty acid composition of various tissues of the marmoset monkey (Callithrix jacchus) after different lipid supplemented diets. Comp. Biochem. Physiol. 101A: 387-393.

CHIVERS, D.J. 1998. Measuring food intake in wild animals: Primates. Proc. Nutr. Soc. 57: 321-332.

CLAPP, N.K.; TARDIF, D.S. 1985. Marmoset husbandry and nutrition. Dig. Dis. Sci. 30:17S-23S.

CORREA, H.K.M.; COUTINHO, P.E.G.; FERRARI, S.F. 2000. Between-year differences in the feeding ecology of highland marmosets (Callithrix aurita and Callithrix flaviceps) in southeastern Brazil. J. Zool. 252:421-427.

DIGBY, L. 1998. Vertebrate predation in common marmosets. Neotrop. Primates 6:124-125.

DIXON, A. 1982. The owl monkey Aotus trivirgatus. Págs.71-113. En: Herdn, J.F (ed.). Reproduction in the new world primates. The zoologist Society of London MTP Press. London.

ELY, A.; FREER, A.; WINDLE, C.; RIDLEY, R.M. 1998. Assessment of cage use by laboratory-bred common. Lab. Anim. 32:427-433.

FAJARDO, A. 1988. Anotaciones sobre el comportamiento alimenticio de Cebuella pygmaea (Spix, 1824) en cautividad (Primates: Callitrichidae). Trianea (Act. Cient. Tecn. INDERENA) 1: 119-122 (1988).

FLURER, C.I.; ZUCKER, H. 1989. Ascorbic acid in a New World monkey family: species difference and influence of stressors on ascorbic acid metabolism. Z. Ernahrungswiss. 28:49-55.

GARBER, P.A. 1992. Vertical clinging, small body size, and the evolution of feeding adaptations in the Callitrichinae. Am. J. Phys. Anthrop. 88:469-482. 
GORE, M.A.; BRANDES, F.; KAUP, FJ.; LENZNER, R.;. MOTHES, T.; OSMAN, A.A. 2001. Callitrichid nutrition and food sensitivity. J. Med. Primatol. $30: 1-6$.

HANNAFORD, G. 1996. Feeding bowl height preferences in captive common marmosets (Callithrix jacchus jacchus). Australian Primatology 11:5-13.

HERNÁNDEZ-CAMACHO, J.; COOPER, R.W. 1976. The nonhuman primates of Colombia. pp. 35-69. En: Thorington, R.W.Jr.; Heltne, P.G. (eds.), Neotropical Primates. Field Studies and Conservation. National Academy of Sciences, Washington, DC.

HLADIK, C.M.; HLADIK, A.; BOUSSET, J.; VALDEBOUZE, P., VIROBEN, G.; DELOT-LAVAL, J.1971. Le regime alimetaire des primates de líle de barro Colorado (Panamá). Folia Pirmatol. 16:85-122.

IPS. 2006. International guidelines for the acquisition, care and breeding of nonhuman primates. The Captive Care and Breeding Committee International Primatological Society.

KIRKWOOD, J.D.; EPSTEIN, M.A.; TERLECKI, A.J. 1983. Factor in fluencing population growth of colony of cotton-top tamarins. Lab. Anin. Sci. 17:35-41.

KIRKWOOD, J.D. 1983. Effcts of diet on health, weigth and litter size in captive cotton-top tamarins Saguinus oedipus. Primates 24(4):515-520.

LASKA, M.; CARRERA-SÁNCHEZ, E.; RODRÍGUEZ-LUNA, E. 1996. Gustatory thresholds for foodassociated sugars in the spider monkey (Ateles geoffroyi). Am. J. Primatol. 39: 189-193.

LASKA, M.; CARRERA-SÁNCHEZ, E.; RODRÍGUEZ-LUNA, E. 1998. Relative taste preferences for food-associated sugars in the spider monkey (Ateles geoffroyi). Primates 39: 91-96.

LASKA, M.; HERNANDEZ-SALAZAR, LT.; RODRIGUEZ-LUNA, E. 2000.Food Preferences and Nutrient Composition in Captive Spider Monkeys, Ateles geoffroyi. International Journal of Primatology, 21(4):671-683.

LASKA, M.; HERNÁNDEZ-SALAZAR, L.T., RODRÍGUEZ-LUNA, E.; HUDSON, R. 2000. Gustatory responsiveness to food-associated acids in the spider monkey, Ateles geoffroyi. Primates 41: 175-183.

LASKA, M.; SCHEUBER, H.P.; CARRERA-SÁNCHEZ, E.; RODRÍGUEZ-LUNA, E. 1999. Taste difference thresholds for sucrose in two species of nonhuman primates. Am. J. Primatol. 48: 153-160.

MERRIT, P.A.Jr. 1976. The owl monkey Aotus trivirgatus: Husbadry, behavior and breeding. Zoological Garden Park. Lincoln Park. Chicago, USA. 
MILTON, K. 1998. Physiological ecology of howlers (Alouatta): Energetic and digestive considerations and comparison with the Colobinae. Int. J. Primatol. 19: 513-548.

NAPKA, J.; MORIN, M. 1979. Open formula ingredients diets for non human primates. Academic Press. Washuington D.C, USA.

NEYMAN, P. 1976. Aspects of ecology and social organization of free-ranging cotton -top tamarins ( $S$. oedipus) and the conservation status species. Págs. 39-71. En: Kleiman, D.K. (ed.). The biology and conservation of Callitrichidae. Smithsonian Inst. Press. Washington, USA.

NICKEL, D.A.; HEYMANN, E.W. 1996. Predation on Orthoptera and other orders of insects by tamarin monkeys, Saguinus mystax mystax and Saguinus fuscicollis nigrifrons (Primates: Callithrichidae), in north-eastern Peru. J. Zool. Lond. 239:799-819.

NRC-NATIONAL RESEARCH COUNCIL. 1978. Nutrient Requirements of Nonhuman Primates. Printing and Publishing Office, National Academy of Sciences, Washington, D.C.

POOLE, T.B. 1990. Environmental enrichment for marmosets. Animal Technology, 41:81-86.

POWER, M.L.; OFTEDAL, O.T. 1996. Differences among captive callitrichids in the digestive responses to dietary gum. Am. J. Primatol. 40:131-144.

RATHBUN, G.B.; GACHE, M.J. 1977. The status of Aotus trivirgatus in Argentina. Centro Argentino sw Primates and Natinal Isnstitute of Helath Report. Washington. D.C.

RICHARD, A.F. 1985. Primate diets: Patterns and principles. pp. 163-205. En: Richard, A.F. (ed.), Primates in Nature. Freeman, W.H. New York, USA.

ROSENBERGER, A.L. 1992. Evolution of feeding niches in New World monkeys. Am. J. Phys. Anthrop. 88:525-562.

SANDERSON, I. 1957. The monkey kingdom. John Murray Ed. London.

SOINI, P. 1977. Informe sobre estudio primatológico: Ecología y dinámica poblacional de Cebuella pygmaea (Primates: Callitrichidae). 30 pp (mimeografiado). Iquitos, Perú.

SOINI, P. 1982. Ecolgy and population dynamics of the pygmy marmoset, Cebuella pygmaea. Folia Primatologica 39:1-21. 
OFTEDAL. O. 1998. Growth variation in common marmoset monkeys (Callithrix jacchus) fed a purified diet: relation to caregiving and weaning behaviors. Lab. Anim. Sci. 48:264-269.

TERBORGH J. 1983. Five New World Primates. A Study of Comparative Ecology. Princeton University Press, Princeton, NJ.

TOWNSEND, W.R. 1999. An observation of carnivory by a captive pygmy marmoset (Cebuella pygmaea). Neotrop. Primates 7:75-76.

UMAÑA, J.; RAMIREZ, J.; ESPINOSA, C.; SABOGAL, C. 1984. Primates no humanos para investigación biomédica, establecimiento, adaptación y mantenimiento de Aotus lemurinus griseimembra. Bol. of Sanit. Panam. 97 (11):44-53.

WRIGTH, P.C. 1978. Home range, activity pattern and agonistic encouters of a group of nigth monkey (Aotus trivirgatus) in Perú.Folia Primatol. 29:43-55. 